\title{
Indigenous access barriers to health care services in London, Ontario
}

\section{The Engaging for Change Improving Health Services for Indigenous Peoples qualitative study}

\author{
Stephanie McConkey
}

Faculty Reviewer: Lloy Wylie, PhD, MA (Department of Psychiatry)

\section{ABSTRACT}

Introduction: Indigenous peoples in Canada suffer higher rates of health inequalities and encounter a number of health services access barriers when compared to their non-Indigenous counterparts. Indigenous peoples experience social and economic challenges, cultural barriers, and discrimination when accessing mainstream health services.

Methods: In London, Ontario, 21 interviews and 2 focus groups $(n=25)$ with service providers were completed, each session spanning approximately 1 to 1.5 hours. Interviews were voice recorded and transcribed verbatim. Themes were identified using NVIVO 10 software.

Findings: Approximately 2 to $5 \%$ of clients are Indigenous in hospital-based services. There are a number of social factors that influence whether Indigenous peoples access health services. Indigenous peoples do not have access to adequate pain medications because physicians are reluctant to provide Indigenous patients with pain medications due to common perceptions of addiction. Indigenous peoples also have barriers accessing a family physician because physicians are reluctant to take on new patients with complex health needs.

Conclusion: Systemic discrimination is still alive in the health care system; therefore, there is a need for cultural safety training among physicians to increase awareness of access barriers and challenges that many Indigenous patients face when seeking health care.

\section{INTRODUCTION}

Indigenous peoples (First Nations, Inuit and Métis) face higher rates of adverse health issues than non-Indigenous people in Canada. Despite the high prevalence of respiratory and circulatory diseases, infectious diseases, injuries, obesity, diabetes, and some cancers, ${ }^{1}$ Indigenous peoples do not receive adequate health care and/or do not like to access conventional medical services because of common experiences of discrimination and racism, delivery of poor quality health care, and other access barriers. ${ }^{2-9}$

Research has shown that Indigenous peoples do use traditional Indigenous approaches to medicine, however, most Indigenous peoples have to seek conventional medicine because that is all that they have access to due to issues of funding and availability of these types of services. ${ }^{7,10}$

With the increase in urbanization among Canada's Indigenous population, these issues have been more rapidly affecting the urban Indigenous population. This is problematic because today more than half of Canada's Indigenous population live in urban settings. ${ }^{11}$ A main explanation for the increase in urbanization among this population is the need to relocate in order to access a range of health and social services. ${ }^{12}$ Those that do not relocate into the city often have to travel far distances to access health and social services in an urban area. ${ }^{8,13}$ Unfortunately, there are a number of challenges that Indigenous peoples face as a result of accessing mainstream services. Some of these challenges include financial challenges, transportation barriers, housing, racism and cultural barriers. ${ }^{13}$ The primary goal of this study was to identify the main health service inequalities that Indigenous peoples experience in an urban setting through exploring the attitudes, knowledge and skills of health care providers that work in hospital and/or community-based health centres.

\section{METHODS}

The Engaging for Change: Improving Health Services for Indigenous Peoples project received research ethics approval from both Western University and Lawson Health Research Ethics Boards. A literature review and discussions with local Indigenous health leaders were carried out to determine priority health research areas for the Indigenous population in London, Ontario prior to commencement of qualitative interviews with health care practitioners. From August 2015 to June 2016, 21 one-on-one interviews and 2 focus group interviews ( 2 participants per focus group) were completed, each interview session spanning approximately 1 to 1.5 hours. Interviewees included physicians, nurses, social workers, patient navigators, patient experience specialists and individuals in a leadership position (eg Department Director) working in a range of health care settings, such as hospitals, community health centres and specialized clinics. Inclusion criteria for participants were those working in a health care setting in one of the five priority services areas (emergency, mental health, maternity, diabetes and cancer) within the London and surrounding area.

Written consent was obtained prior to commencement of a structured interview or focus group. All participants were asked 20 in-depth questions regarding their experiences working with or providing health care to Indigenous peoples. Focus group participants would take turns responding to the interview questions. Questions touched on topics including access barriers, positive and negative experiences, policies and protocols surrounding Indigenous health, and communication, coordination and collaboration with other local community health and social services. A full-list of 
interview questions can be found in Appendix A. Qualitative interviews were voice recorded and transcribed verbatim by qualified research assistants. Afterwards, coding was completed using NVIVO 10 Software to identify the main emerging themes.

\section{RESULTS}

\section{Patient Population}

Themes of qualitative interviews identified that hospital based programs noted that only 2 to $5 \%$ of patients and clients were Indigenous peoples, however, respondents identified that the percentage of Indigenous patients was higher in diabetes-related programs. Additionally, physicians believe that Indigenous peoples do not properly articulate their health issues to physicians, which could play a role in the low percentage of Indigenous patients and clients within the system.

\section{Social Determinants of Health}

Health care professionals understood that there are a number of reasons why Indigenous peoples may be reluctant to access health care services. While some physicians did express the need for patients to take individual responsibility, the majority of respondents acknowledged the social determinants of health that may hinder access to health care. Social determinants of health that were discussed in the interviews included high levels of poverty and unemployment, low education levels, and other social issues that may influence an individual's health and wellbeing. Transportation to health facilities was believed to be the main access barrier among Indigenous peoples living in rural and/or remote communities, such as reserves. For example, one physician responded,

"I would say that people living in the community that don't have the resources or the support to come to London. I think they definitely struggle more with accessing services" (AB1OP).

Another commonly emerging theme was that physicians believed that Indigenous peoples suffer from more co-morbidities than their non-Indigenous counterparts and admitted that there may be an issue with physicians being reluctant to take on patients with complex health care needs. A physician noted,

"so there is a new doc who you know, is setting up a shingle in town and a new office and they interview patients and they go 'wow, you are pretty complicated I don't really want to get involved with you' and so that exacerbates the whole doctor shortage" (H23P).

This finding demonstrates the shortage of family doctors, but highlights the particular challenges for people with complex health needs, which is more common among Indigenous peoples. ${ }^{14-16}$

\section{Racism and Discrimination}

A number of participants shared that they were unaware of traditional Indigenous practices and perspectives of health and wellbeing, however they acknowledged that these practices differed from conventional approaches to medicine. It was also identified that Indigenous practices and perspectives of health and wellbeing were not valued in the health care system. Findings also suggest that physicians are reluctant to prescribe pain medications to Indigenous peoples because of common perceptions of addictions and drug abuse. For example, one physician shared his beliefs about systematic discrimination within the health care system as,

"I would think that there are, that on our side there are some frequency of notions that they are all drug seekers. Which clearly isn't true, but I think that if you lined up 10 patients with the same condition and how much pain meds would you give this person if they were Aboriginal, I would bet less, because the fear would be that they are there looking for pain meds to abuse or sell, so I think that as a group they are linked" (A04P).

This response signifies that physicians are aware that there are still discriminatory behaviours among physicians that may affect how an Indigenous patient is cared for.

Physicians also raised the issue that many of their Indigenous clients discontinue services. Physicians believe that this theme may be correlated to poor follow-up and/or lack of appropriate referrals, however, research has shown that it could be strongly related to the experiences of discrimination and racism that Indigenous peoples face within the system. ${ }^{3,7}$ Another explanation for lack of follow-up could be jurisdictional challenges. For example,

"I had several unfortunate patient situations when I worked in the emergency department specific to Aboriginal patients and that was around getting appropriate follow-up care and access to follow-up care because there were difficulties in having community care access get onto the reserve to provide care there. There were some barriers around that" (AO3L).

This response provides evidence that there are jurisdictional issues that Indigenous peoples living on-reserve face which negatively influences the types of follow-up care they have access to.

\section{DISCUSSION}

The findings from this research show that there is a high need for mandatory Indigenous cultural competency and safety training among all providers working in the health care system so that they will be able to provide more culturally relevant and safe care. Research has shown that there is a lack of culturally appropriate health services, ${ }^{4,17-18}$ and our findings align with research that suggests that Indigenous peoples are still facing systemic discrimination when accessing mainstream health care services and programs. ${ }^{3}$ Racial misconceptions can strongly influence an individual's health and wellbeing, and make them reluctant to access health care as a result. ${ }^{3-7}$ Therefore, health provider training should take on the biases and stereotypes that lead to the provision of poor standards of care for Indigenous patients. Participation in cultural safety training would be a means of reducing racist and discriminatory perceptions 
about Indigenous peoples that are widespread within the health care system. Additionally, cultural awareness will be beneficial for care providers to support their understanding of the different perspectives of health and wellbeing among Indigenous peoples. Such knowledge would facilitate improved patient support and better communication and coordination with community-based services.

Supports need to be put in place to facilitate access to health care services. It is clear that transportation is definitely a barrier to those who do not reside in the city, because reserves do not have the same structural support that any given city would in regards to health care. Therefore, individuals need to travel to the city to get adequate access to health care ${ }^{8,13}$ Funding for transportation for those living in rural settings would provide Indigenous patients and families the ability to access health services within and out of their community.

More awareness and communication between hospital programs and community supports (ie. Southwestern Ontario Aboriginal Health Access Centre, N'Amerind Friendship Centre, etc.) are needed to ensure patients needs are being met and proper referrals are being completed. Additionally, further research on continuity of care needs to be done to understand why it is lacking among the Indigenous population in London, Ontario.

Limitations of this study is that there was a small sample size $(n=25)$ and only health care provider perspectives were included in the research at this time. Additionally, ethnicity data is not collected in hospital-based services, therefore the number of Indigenous patients was an estimation based on the respondents' recollection.

\section{CONCLUSION}

In order to improve health access for Indigenous peoples, health care providers need to be aware of the issues and challenges that many Indigenous peoples have surrounding primary health care, most importantly the social determinants of health. Physicians working in diabetes-related services should be aware of the higher percentage of Indigenous peoples in these services, and proper training should take place to ensure cultural needs are being met. It is evident that racism and discrimination are still alive in the health care system and it has strong influences on the health and wellbeing of Indigenous peoples. The next steps for this research is to understand the impact of cultural safety training on health care workers and to determine what other institutional supports are needed to transform practice and patient experiences. In addition, the project aims to understand patient perspectives and needs to identify how to ensure appropriate care is provided when they access hospital and community-based programs in London, Ontario.

\section{APPENDIX A - Engaging For Change: INTERVIEW QUESTIONS}

1. What is your position in your organization/unit and what type of services do you provide?

2. Approximately what percentage of your patients/clients are Aboriginal? Has their numbers changed recently?

3. How would you describe the current condition of Aboriginal access to health care in your service? Do all Aboriginal groups have the same level of access? What are the differences?

4. Briefly describe an experience with an Aboriginal patient that you feel was positive. Do your best to make clean and reflect upon what you think made it a positive experience.

5. Briefly describe an experience with an Aboriginal patient that you feel was negative. Do your best to make clean and reflect upon what you think made it a negative experience.

6. What do you think are the greatest challenges in providing care to Aboriginal patients?

7. What do you think are the greatest challenges facing Aboriginal patients?

8. Are their specific services offered to Aboriginal people? Which? Do you provide culturally appropriate resource materials? System navigation supports?

9. How do you provide for Aboriginal specific needs (eg. Spiritual practices etc.) in your institution (in the health care system)?

10. Are you aware of alternative healing practices that Aboriginal people use? If yes, are systems in place to facilitate incorporation of alternative practices into patient care plans?

11. What are some ways you think would ensure the provision of culturally sensitive services for Aboriginal patients?

12. What supports would you need to help you provide more culturally sensitive services for Aboriginal patients?

13. Are there Aboriginal employees in your organization?

14. Are your employees skilled in intercultural communication, Aboriginal cultural sensitivity? Do you provide on-the-job training in cultural competence/sensitivity/safety? If yes, how often?

15. Are you aware of any policies/protocols for facilitating inclusion of Aboriginal perspectives in or improving access to health services?

16. What types of policies/protocols could improve access and experiences of Aboriginal patients in the health care system? 17. Are you aware of community resources that could enhance both understanding of and service delivery with Aboriginal patients? 18. Which organizations do you collaborate with around health service provision for Aboriginal patients? Around referrals and discharge?

19. Do you think there is a need for improving coordination between your program and other services? What are some concrete examples of coordination that would improve continuity of care? 20. Does your institution engage the Aboriginal population for program feedback? If so, how is this done? 


\section{REFERENCES}

1. Mikkonen J, Raphael D. The social determinants of health: the Canadian facts. Toronto: York University School of Health Policy and Management; 2010. Chapter 13, Aboriginal Status; p. 41-3.

2. Adelson N. The embodiment of inequity: Health disparities in Aboriginal Canada. Canadian Journal of Public Health/Revue Canadienne de Sante'e Publique. 2005 Mar;96: S45-61.

3. Allan B, Smylie J. First Peoples, second class treatment: The role of racism in the health and well-being of Indigenous peoples in Canada. Toronto: the Wellesley Institute; 2015.

4. Benoit C, Carroll D, Chaudhry M. In search of a healing place: Aboriginal women in Vancouver's downtown eastside. Social science \& medicine. $2003 \mathrm{Feb} ; 56(4): 821-33$

5. Browne AJ, Fiske JA. First Nations women's encounters with mainstream health care services. Western Journal of Nursing Research. 2001 Mar;23(2):126-47.

6. Lallo S. Prenatal care through the eyes of Canadian Aboriginal women. Nursing for Women's Health. 2014 Feb;18(1):38-46.

7. NAHO [Internet]. What First Nations people think about their health and health care: National Aboriginal Health Organization's public opinion poll on Aboriginal health and health care in Canada. National Aboriginal Health Organization; 2003 July [cited 2017 Mar 28]. Available from: http://www.naho.ca/documents/fnc/english/FNC_SummaryofNAHOPoll.pdf.

8. Reading CL, Wien F. Health inequalities and the social determinants of Aboriginal peoples' health. Prince George, BC: National Collaborating Centre for Aboriginal Health; 2009.

9. Richmond CA, Ross NA. The determinants of First Nation and Inuit health: A critical population health approach. Health \& Place. 2009 Jun;15(2):403-11.

10. Waldram JB, Herring A, Young TK. Aboriginal health in Canada: Historical, cultural, and epidemiological perspectives. Toronto: University of Toronto Press; 2006.

11. Norris MJ, Clatworthy S, Peters E. Indigenous in the city: contemporary identities and cultural innovation. Vancouver: UBC Press; 2013. Chapter 1, The Urbanization of Aboriginal Populations in Canada; $p$. $32-43$.

12. Newhouse D, Peters EJ. Not strangers in these parts: Urban Aboriginal peoples. Policy Research Initiative; 2003.

13. Place $J$. The health of Aboriginal people residing in urban areas. Prince George, BC: National Collaborating Centre for Aboriginal Health; 2012.

14. Gracey M, King M. Indigenous health part 1: determinants and disease patterns. The Lancet. 2009 Jul 10;374(9683):65-75.

15. First Nations Information Governance Centre (FNIGC). First Nations Regional Health Survey (RHS) 2008/10: National report on adults, youth and children living in First Nations communities. Ottawa: FNIGC; 2012.

16. Ring I, Brown N. The health status of indigenous peoples and others. BMJ. 2003 Aug 23;327(7412):404-5.

17. Browne AJ, Smye V. A post-colonial analysis of healthcare discourses addressing aboriginal women. Nurse Researcher. 2002 Apr;9(3):28-41.

18. Browne AJ, Smye VL, Varcoe C. The relevance of postcolonial theoretical perspectives to research in Aboriginal health. Canadian Journal of Nursing Research. 2005 Dec;37(4):16-37.

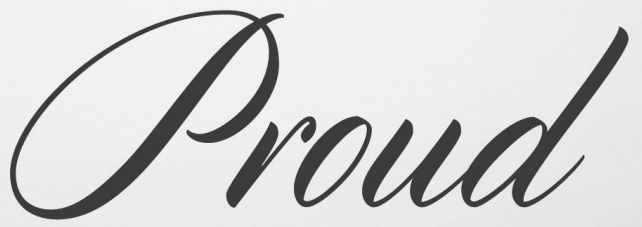

to be a part of your medical journey

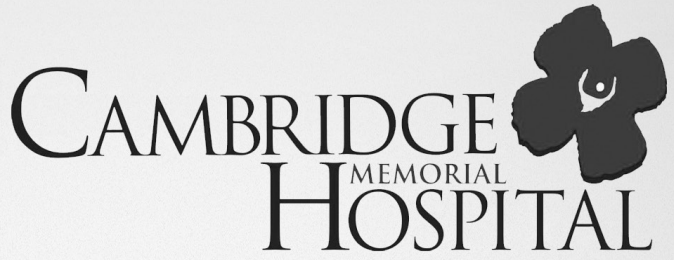

